\title{
Relación entre la regulación emocional y la autoestima
}

\author{
NÚRIA BALAGUER PICH \\ al313720@uji.es \\ MARTín SÁNCHEZ GÓMEZ \\ martin.sanchez@uji.es \\ AZUCENA García Palacios \\ azucena@uji.es
}

\section{Resumen}

Introducción: Autoestima y regulación emocional son constructos psicológicos relacionados con la salud mental. El presente estudio pretende explorar la relación existente entre estos dos constructos. Objetivo: Analizar la relación entre la autoestima y distintas dimensiones de la regulación emocional y conocer cuáles tienen más relación con la autoestima. Método: Cien personas, entre 20 y 30 años, de la población general participaron en el estudio (51 mujeres y 49 hombres). Fueron evaluadas a través del Cuestionario de Autoestima de Rosenberg (Rosenberg, 1965) y la Escala de Dificultades en la Regulación Emocional (DERS, Gratz y Roemer, 2004). Resultados: Los resultados muestran una correlación significativa entre la autoestima y las subescalas del DERS: Atención, Claridad, Aceptación, Funcionamiento y Regulación. El análisis de regresión reveló que las dimensiones más relacionadas con la autoestima fueron la Aceptación y la Atención. Conclusión: Es esperable encontrar una relación entre la capacidad de regular las emociones y un constructo que supone una valoración positiva de uno mismo, la autoestima. En concreto, la capacidad de prestar atención a las emociones y aceptarlas podrían ser aspectos fundamentales en la construcción de un autoconcepto y una valoración de sí mismo positivos. En investigaciones futuras se propone estudiar en profundidad estas relaciones con el fin de poder realizar recomendaciones sobre qué aspectos de la regulación emocional trabajar con el fin de potenciar autoestima y bienestar.

Palabras clave: Autoestima, regulación emocional, emociones, aceptación, salud mental.

\section{Abstract}

Introduction: Self-esteem and emotional regulation are psychological constructs relate to mental health. The current research pretends to explore the relation between these two constructs. Objective: Analyze the relation between self-esteem and differents dimensions of emotional regulation, and knowing which have more relation with the self-esteem. Method: one hundred people from the general population participated in this study (51 women and 49 men), with ages between 20-30 years were evaluated through the Rosenberg self-esteem questionnaire (Rosenberg, 1965) and Scale of dif- 
ficulty in emotional regulation (DERS, Gratz \& Roemer, 2004). Results: The results show a significant correlation between self-esteem and the subscales of the DERS: attention, clarity, acceptance, operation and regulation. Regression analysis revealed that most related to self-esteem dimensions were acceptance and attention. Conclusion: It is expected to find a relationship between the ability to regulate emotions and a construct that implies a positive evaluation of one's own, the self-esteem. In particular, the ability to pay attention to emotions and accept could be key aspects in constructing a positive self-concept and self-assessment. In future research it is proposed to study in depth these relationships in order to be able to make recommendations about which aspects work in emotional regulation in order to enhance self-esteem and well-being.

Key Words: Self-esteem, emotional regulation, emotions, acceptance, mental health.

\section{Introducción}

Como indican recientes investigaciones, el modelo transdiagnóstico es una propuesta multidimensional que aborda el estudio y tratamiento de los trastornos emocionales en base a dimensiones psicopatológicas comunes a distintos trastornos (Kirszman y del Carmen Salgueiro, 2015).

Existen ya diversos modelos transdiagnósticos que están proporcionando mejoras en la comprensión y tratamiento de distintos trastornos. En este trabajo mencionaremos dos modelos que consideramos importantes, ya que en ellos se indican los constructos objetivo de este trabajo, autoestima y regulación emocional. Estos modelos son el modelo transdiagnóstico para los trastornos alimentarios de Fairburn (Fairburn, Cooper y Shafran, 2003) y el modelo transdiagnóstico para los trastornos emocionales de Brown y Barlow (2009). Las investigaciones más recientes han coincidido en encontrar diversos problemas de regulación emocional en la población clínica. En concreto, estos problemas se han encontrado en pacientes con trastornos depresivos, en la mayoría de los trastornos de ansiedad y en los trastornos de la personalidad, sobre todo en el trastorno límite de personalidad. Las personas que sufren estos trastornos presentarian déficits de regulación emocional, es decir, desregulación afectiva. Estos avances en las propuestas de clasificación están dando lugar a perspectivas novedosas en el tratamiento de los trastornos emocionales, como el protocolo unificado para el tratamiento transdiagnóstico de los trastornos emocionales (Barlow y cols., 2010), el cual propone que hay evidencia que atestigua la amplia comorbilidad diagnóstica entre los trastornos de ansiedad y estado de ánimo. Estudios longitudinales indican que la covariación temporal de estos trastornos puede explicarse por cambios en Neuroticismo/Inhibición conductual (N/IC) y, en algunos casos, niveles iniciales de N/IC son predictivos en el curso temporal de los trastornos emocionales. La superposición de los trastornos de ansiedad y estado de ánimo del DSM-IV (APA, 2000) es una fuente frecuente de falta de fiabilidad diagnóstica (por ejemplo, superposición temporal de las características comunes del trastorno de ansiedad generalizada y trastornos del ánimo, situación específica de ataques de pánico en el trastorno de pánico y fobia específica). Aunque las propuestas dimensionales pueden abordar algunos inconvenientes asociados a la nosología del DSM (por ejemplo, inadecuada evaluación de las diferencias individuales en la severidad de la enfermedad), estas propuestas no reconcilian los problemas 
clave en la clasificación actual, como una modesta fiabilidad y una alta comorbilidad (Brown y Barlow, 2009).

Déficits en la regulación emocional son comunes a estos trastornos y los pacientes con trastornos emocionales habitualmente utilizan estrategias desadaptativas de regulación emocional que contribuyen al mantenimiento de los síntomas.

También existen protocolos transdiagnósticos en otros trastornos como los trastornos alimentarios (Fairburn, 2008). Este protocolo se refiere a los procesos psicopatológicos que son responsables de la persistencia de graves trastornos de la alimentación. Se desarrollan dos líneas argumentales independientes pero interrelacionadas. Uno es que se amplíe la teoría basada en la evidencia principal del mantenimiento de los trastornos alimentarios, la teoría cognitiva conductual de la bulimia nerviosa. En concreto, se propone que en ciertos pacientes, uno o más de cuatro procesos interactúan con el trastorno base y que cuando esto ocurre es un obstáculo para el cambio. Los procesos de mantenimiento adicionales son el perfeccionismo clínico, la baja autoestima, la intolerancia a las emociones y las dificultades interpersonales. La segunda línea de argumentación es que en el caso de trastornos alimentarios compartidos, pero distintivos, algunas características clínicas tienden a ser mantenidas por procesos psicopatológicos similares. Por consiguiente, se sugiere qu e esos mecanismos comunes están implicados en la la bulimia nerviosa, la anorexia nerviosa y los trastornos atípicos. Juntas, estas dos líneas de argumentación llevan a proponer una nueva teoría transdiagnóstica del mantenimiento de toda la gama de trastornos alimentarios, una teoría que abarca una gama más amplia en mantener los mecanismos de la teoría actual sobre la bulimia nerviosa (Fairburn, 2003).

En este trabajo estamos interesados en estudiar dos constructos relevantes en estos modelos transdiagnósticos, la autoestima y la regulación emocional.

En la literatura científica revisada, es posible apreciar la inexistencia de un acuerdo en relación al concepto de regulación emocional y autoestima. Sin embargo, hemos considerado la definición de Bonet acerca de la autoestima y la definición de Thompson sobre la regulación emocional, como las más cercanas a nuestra perspectiva de estudio. Así pues, Bonet (1997) define autoestima como un "sentimiento valorativo dirigido a nosotros mismos y nuestro conjunto de rasgos construido a través de la influencia de múltiples factores y experiencias a lo largo de la vida»(Bonet, 1997), y Thompson (1994), define regulación emocional como el «proceso de iniciar, mantener, modular o cambiar la ocurrencia, intensidad o duración de los estados afectivos internos y los procesos fisiológicos, a menudo con el objetivo de alcanzar una meta» ( $p$. 106). Esta definición se considera como base, ya que permite conceptualizar la regulación emocional como un proceso que ayuda al ser humano a manejar sus estados emocionales, para lo que puede utilizar distintos tipos de estrategias que conducen a ese objetivo.

Nuestro objetivo es demostrar que estos dos constructos correlacionan y podemos categorizarlos como dimensiones que poseen diferentes trastornos emocionales. La hipótesis del estudio es la existencia de correlación negativa entre la autoestima y las dificultades en regulación emocional. Es decir, demostrar que la autoestima que posee una persona y las dificultades de gestión emocional que pueda tener son dos factores que correlacionan entre sí e influyen en la salud mental, puesto que presentar dificultades en regulación emocional junto con una baja autoestima podrían favorecer el inicio de trastornos emocionales como depresión o trastornos de ansiedad, así como el mantenimiento de éstos. Profundizando en este estudio correlacional, veremos si algunas subdimensiones de la regulación emocional correlacionan más que otras con la autoestima mediante un análisis de regresión lineal paso a paso.

Las subdimensiones de la escala DERS son habilidades conceptualizadas como elementos centrales para la regulación emocional, y las altas puntuaciones en estas habilidades mostrarán en los participantes, dificultades en la gestión emocional. Los diferentes aspectos 
del proceso de la regulación emocional en los que pueden existir dificultades son: Descontrol emocional, Interferencia cotidiana, Desatención emocional, Confusión emocional y Rechazo emocional. Por contra, estas cinco subdimensiones tienen su lado opuesto: Control emocional, Funcionamiento emocional. Atención emocional, Claridad emocional y Aceptación emocional (Hervás y Jódar, 2008).

\section{Método}

\section{Participantes}

Como criterios de inclusión se establecieron: a) no estar o haber estado en tratamiento psicológico o psiquiátrico, de este modo nos aseguramos de no contar con población clínica; b) Con respecto al rango de edad, se requirió tener una edad comprendida entre 20 y 30 años.

Después de aplicar los criterios de selección, la muestra inicial de 168 personas se redujo a 100 participantes, de los cuales 51 eran mujeres. La media de edad fue de 22,1 años con un $90 \%$ de población universitaria, concretamente estudiantes de la Universidad Jaume I debido a la accesibilidad. Los objetivos y fines del estudio fueron presentados durante el primer contacto con la población.

\section{Instrumentos de evaluación}

La regulación emocional fue evaluada mediante la escala DERS (Escala de Dificultades en la Regulación Emocional (DERS, Difficulties in Emotion Regulation Scale) (Gratz y Roemer, 2004), que cuenta con un alto índice de confiabilidad ( $\alpha=.93)$, y sus cinco subescalas (atención-desatención; claridad-confusión; aceptación-rechazo; funcionamiento-interferencia; regulación-descontrol) para evaluar diferentes aspectos de la desregulación emocional a través de una escala Likert de 5 puntos (de «Casi nunca / 0-10\% de las veces» a "Casi siempre / 90$100 \%$ de las veces»). La escala DERS permite medir diferentes aspectos de la regulación desadaptativa, aspectos considerados clínicamente relevantes, y que al mismo tiempo abarcan las dificultades funcionales y déficits más importantes que pueden presentarse en el transcurso del procesamiento emocional de un suceso.

En cuanto a la autoestima, fue medida a través del Cuestionario de Autoestima de Rosenberg (RSQ; Rosenberg, 1965) ( $\alpha=.92$; Fernández-Montalvo y Echeburúa, 1997), que evalúa el sentimiento de satisfacción que una persona tiene sobre sí misma a través de una escala Likert de 4 puntos.

\section{Procedimiento}

El procedimiento se inició estableciendo los criterios de selección muestral para obtener una población que cumpliera con las características de planteadas para el estudio. Después se procedió a la recogida de datos. Esta se alargó alrededor de un mes, los participantes no obtuvieron ninguna retribución y su colaboración fue totalmente anónima para cumplir con la confidencialidad. La totalidad de los test se realizaron de manera online. 
Los instrumentos de medida utilizados fueron transformados a formato online a través del Google Forms para cuestionarios y se difundieron por diferentes canales de comunicación como la mensajería móvil, las redes sociales y el correo electrónico.

Análisis de datos

Una vez obtenidas las respuestas se procedió a almacenar los datos y posteriormente a su análisis mediante el programa estadístico SPSS (versión 24.0). Tras ello, efectuamos las correspondiente correlaciones bivariadas mediante la $r$ de Pearson y análisis de regresión paso a paso (stepwise).

\section{Resultados}

En primer lugar, cuando analizamos mediante la correlación de Pearson las diferentes subescalas del DERS y la escala de autoestima de Rosenberg, observamos que las correlaciones (ver Tabla 1) eran significativamente altas en sentido negativo. Este resultado nos mostró que dificultades en atención emocional, claridad emocional, aceptación de las emociones, funcionamiento y, por último, dificultades en la regulación emocional, tienen una relación negativa significativa con el nivel de autoestima.

Tabla 1

Correlación de las variables de estudio (Pearson)

\begin{tabular}{cccccc}
\hline Variable & Atención & Claridad & Aceptación & Funcionamiento & Regulación \\
\hline Autoestima &,$- 547^{* *}$ &,$- 469^{* *}$ &,$- 583^{* *}$ &,$- 327^{* *}$ &,$- 463^{* *}$ \\
\hline
\end{tabular}

** La correlación es significativa en el nivel 0,01

En segundo lugar, teniendo en cuenta los resultados obtenidos anteriormente, se llevó a cabo un análisis de regresión Stepwise en el que se incluyeron las variables del DERS para evaluar el valor predictivo de las dimensiones de regulación emocional con respecto a la autoestima. En la tabla 2 se ofrecen los resultados de dicho análisis. Tras realizar el análisis de regresión sobre las variables implicadas, se obtuvo que las dos que mayor relación tenían con la autoestima fueron la aceptación y la atención. 
Tabla 2

Análisis de regresión Stepwise

\begin{tabular}{|c|c|c|c|c|c|c|}
\hline Modelo & & \multicolumn{2}{|c|}{$\begin{array}{c}\text { Coeficientes no } \\
\text { estandarizados }\end{array}$} & $\begin{array}{c}\text { Coeficientes } \\
\text { estandarizados }\end{array}$ & $\mathrm{t}$ & $\mathrm{p}$ \\
\hline \multirow{2}{*}{2} & & $\mathrm{~B}$ & Error típico & Beta & & \\
\hline & (Constante) & 42,882 & 1,351 & & 31,731 & 0,000 \\
& DERS: & $-0,378$ & 0,065 & $-0,450$ & $-5,777$ & 0,000 \\
& Aceptación & $-0,666$ & 0,131 & $-0,396$ & $-5,082$ & 0,000 \\
& DERS: & & & & & \\
& Atención & & & & & \\
\hline
\end{tabular}

Variable dependiente: Autoestima

\section{Conclusión}

El objetivo de este trabajo era analizar la relación entre dos constructos, autoestima y regulación emocional, mediante la evaluación de distintas subdimensiones como la claridad emocional (o por contra confusión), la aceptación emocional (o por contra rechazo), la atención a las emociones (o por contra desatención), el funcionamiento emocional (o por contra interferencia), el control emocional (o por contra descontrol) y finalmente, la autoestima. Los resultados obtenidos muestran que todas las subdimensiones relacionadas con las dificultades en la regulación emocional correlacionan de forma significativa y en sentido negativo con la dimensión de autoestima, siendo las subdimensiones de aceptación y atención emocional las que guardan una relación mayor, según indica el análisis de regresión. Ambas subdimensiones son muy importantes para la regulación emocional, ya que están relacionadas con prestar atención a las emociones y no huir de ellas. Nuestros resultados podrían indicar que la aceptación de una esfera tan importante como es la afectividad, nuestras emociones, contribuiría a la aceptación y valoración de nosotros mismos en términos globales, es decir, contribuiría a una autoestima sana.

Nuestros datos indican que existe una gran covariación entre los dos constructos claves del estudio, autoestima y regulación emocional. Tras haber analizado los resultados de forma pormenorizada podemos decir que, se encuentran en consonancia con la hipótesis planteada.

Nos detendremos ahora en explicar más detalladamente cada subdimensión de la regulación emocional, la autoestima, y la relación entre ambos constructos. Por una parte, Gratz y Roemer (2004) definen la atención emocional como la habilidad de atender y entender respuestas emocionales. Esta es la categoría para medir las dificultades en reconocer y darse cuenta de sus propias emociones. Sin embargo, hay algunas definiciones alternativas de atención desde una perspectiva analítica conductual. Por ejemplo, León y Diego (2006) definen atención emocional como una habilidad para experimentar la propia emoción, reconocerla y observarla. Para reducir la brecha en la definición, Caycedo, Gutiérrez, Ascencio y Delgado (2005) plantearon que esta se relaciona con las habilidades de regulación emocional. Por esto, cuando los individuos reconocen las emociones, el contexto donde ocurren y otros repertorios conductuales emitidos para manejar las respuestas emocionales, ellos pueden modular eficazmente sus propios comportamientos. Esto significa que la consciencia de esa atención emocional puede ser un prerrequisito conductual para la regulación emocional, como una habilidad amplia e independiente (Weis, Gratz y Lavender, 2015). Por otra parte, la aceptación 
emocional es la habilidad de experimentar emociones sin intentar controlarlas o cambiarlas. Por lo tanto, hay un estrecho vínculo con la experiencia emocional, por lo que es responsable de alguna parte de las habilidades para regular la emoción. La categoría de no-aceptación (rechazo) emocional hace referencia a la reacción negativa, a las respuestas emocionales de uno mismo y de otros. El rechazo emocional está estrechamente relacionado con la intolerancia al malestar, que se destaca por ejemplo en las personas con trastorno límite de la personalidad. La dificultad en conductas dirigidas a metas implica una interferencia emocional en una acción efectiva hacia una meta, mientras las personas están experimentando emociones negativas. El descontrol o la impulsividad emocional se refiere a los problemas para controlar el propio comportamiento cuando se experimenta una emoción con alta intensidad. Finalmente, la claridad emocional mide las dificultades para diferenciar las emociones mientras estas son experimentadas (Gratz y Roemer, 2004).

El acceso limitado a estrategias de regulación emocional hace referencia a la pobre percepción de habilidades para modular las emociones y conlleva a una estrecha relación con la baja autoestima. Podemos concluir que la íntima relación de la autoestima con la capacidad de gestión emocional convierte a estas dos dimensiones en pilares básicos para poder trabajar en emociones específicas y estrategias de regulación emocional y poder así potenciar el bienestar de las personas. De este modo, las estrategias utilizadas para sentir, expresar y regular nuestras emociones estarán íntimamente ligadas a nuestra autoestima.

La autoestima es otra de las variables que ha demostrado estar relacionada con la regulación emocional (Smith y Petty, 1995; Heimpel y cols., 2002; Setliff y Marmurek, 2002). A través de diferentes investigaciones, se ha demostrado que una baja autoestima está asociada a importantes déficits de regulación afectiva (Hervás y Vázquez, 2006). Por ejemplo, algunos de los síntomas psicopatológicos más preocupantes como las conductas autolesivas o el consumo de sustancias, constituyen, a veces, intentos desadaptativos de regulación emocional (McNally, Palfai, Levine, y Moore, 2003; Gratz, 2003). Dada la relación entre la autoestima y la regulación emocional, es conveniente incluir este constructo en las intervenciones dirigidas a mejorar las estrategias de regulación emocional.

El presente estudio cuenta con algunas limitaciones que nos gustaría destacar. El instrumento de medida DERS, herramienta para conocer dificultades de regulación emocional, es una medida de autoinforme, este tipo de autoevaluación ha generado dudas acerca de su utilidad para evaluar habilidades, ya que son difícilmente medibles a través de preguntas escritas. Sería conveniente combinar los datos de autoinforme con alguna tarea más objetiva en la que la persona fuera expuesta a emociones y tuviera que regularlas. Otra limitación en nuestro estudio es que es meramente correlacional y, por tanto, no podemos establecer relaciones de causa-efecto entre los constructos estudiados.

Entre nuestras líneas futuras pretenden ampliar esta investigación y realizar análisis causales (por medio de paradigmas experimentales) para conocer en qué sentido y de qué forma se relacionan entre ellas estas variables o qué influencias mutuas ejercen entre sí.

\section{Referencias bibliográficas}

Barlow, D. H., Farchione, T. J., Fairholme, C. P., Ellard, K. K., Boisseau, C. L., Allen, L. B., y May, J. T. E. (2010). Unified protocol for transdiagnostic treatment of emotional disorders: Therapist guide. Oxford University Press.

Bonet, J. V. (1997). Sé amigo de ti mismo: manual de autoestima. Santander: Sal Terrae. 
Brown, T. A. y Barlow, D. H. (2009). A proposal for a dimensional classification system based on the shared features of the DSM-IV anxiety and mood disorders: implications for assessment and treatment. Psychological assessment, 21(3), 256.

Caycedo, C., Gutiérrez, C., Ascencio, V. y Delgado, A.(2005). Regulación emocional y entrenamiento en solución de problemas sociales como herramienta de prevención para niños de 5 a 6 años. Suma psicológica, 12(2), 157-173.

Fairburn, C. G., Cooper, Z. y Shafran, R. (2003). Cognitive behaviour therapy for eating disorders: $A$ «transdiagnostic» theory and treatment. Behaviour research and therapy, 41(5), 509-528.

Fairburn, C. G. (2008). Cognitive behavior therapy and eating disorders. Guilford Press.

Gratz, K. L. y Roemer, L. (2004). Multidimensional assessment of emotion regulation and dysregulation: Development, factor structure, and initial validation of the difficulties in emotion regulation scale. Journal of psychopathology and behavioral assessment, 26(1), 41-54.

Hervás, G. y Jódar, R. (2008). Adaptación al castellano de la Escala de Dificultades en la Regulación Emocional. Clínica y Salud, 19(2), 139-156.

Hervás, G. y Vázquez, C. (2006). La regulación afectiva: modelos, investigación e implicaciones para la salud mental y física. Revista de psicología general y aplicada, 59(1-2), 9-36.

Kirszman, D. y Del Carmen Salgueiro, M. (2015). Body image. Mediating dimensions in psychopathology and types of intervention. Revista Mexicana de Trastornos Alimentarios, 6(2), 129-136.

León, R. y Diego, A. (2006). ¿Es explicable la conciencia sin emoción?: una aproximación biológico-afectiva a la experiencia consciente. Revista latinoamericana de Psicologia, 38(2), 361-381.

McNally, A. M., Palfai T. P., Levine, R. V. y Moore, B. M. (2003). Attachment dimensions and drinking-related problems among young adults: The mediational role of coping motives. Addictive Behaviors, 28, 1115-1127.

Muñoz-Martínez, A. M., Vargas, R. M. y Hoyos-González, J. S. (2016). Escala de dificultades en regulación emocional (DERS): Análisis factorial en una muestra colombiana. Acta Colombiana de Psicología, 19(1), 237-248.

Rosenberg, M. (1965). Rosenberg self-esteem scale (RSE). Acceptance and commitment therapy. Measures package, 61, 52.

Thompson, R. A. (1994). Emotion regulation: A theme in search of definition. Monographs of the society for research in child development, 59(2 - 3), 25-52.

Weiss, N. H., Gratz, K. L. y Lavender, J. M. (2015). Factor structure and initial validation of a multidimensional measure of difficulties in the regulation of positive emotions: the DERSpositive. Behavior modification, 39(3), 431-453. 